\title{
REVIEW ARTICLE OPEN Detection of Majorana zero mode in the vortex
}

\author{
Hao-Hua Sun ${ }^{1,2}$ and Jin-Feng Jia ${ }^{1,2}$
}

Majorana zero modes, which behave like Majorana fermions, are quasiparticle excitations in condensed matter systems. They obey non-Abelian statistics, and have been proposed as building blocks of topological quantum computers. They are predicted to exist in the vortex of topological superconductors. In 2012, such a topological superconductor was engineered by depositing topological insulator thin films on top of an s-wave superconductor. Thereafter, several evidences have been reported to prove the Majorana zero modes' existence in the vortex. In this review, by putting all experimental and theoretical results together, we show that these experimental evidences are consistent and they are also strongly supported by the theories, so the existence of Majorana zero mode is firmly established. Moreover, the adjacent Majorana zero modes annihilate when two vortices are close enough, which demonstrate that they have the nature of Majorana fermions. Finally, their potential application in topological quantum computing is discussed.

npj Quantum Materials (2017)2:34 ; doi:10.1038/s41535-017-0037-4

\section{INTRODUCTION}

Majorana fermions (MFs), the elusive particles that are their own antiparticle, were firstly predicted by Ettore Majorana. ${ }^{1}$ Now, their concept is finding ever wider relevance in modern physics, not only in neutrino physics, supersymmetry, and dark matter, but also on some exotic states of condensed matter. ${ }^{2,3}$ The recent effort in searching such a genuine particles focuses on the neutrinoless double beta decay experiment. ${ }^{4}$

Some quasiparticles, which behave like MFs, may emerge as a novel excitation in some condensed matter systems. These Majorana quasiparticles obey non-Abelian statistics and may be used as robust building blocks in quantum computing. ${ }^{5}$ Due to the scientific importance and potential applications in quantum computing, Majorana quasiparticles have attracted lots of attentions recently.

Theoretically, Majorana quasiparticles are predicted to emerge in the vortex core of topological superconductors (TSC) as a zero energy excitation mode called Majorana zero mode (MZM). Unfortunately, there are at present no known materials with this property. In 2008, the search for MZMs took a big step forward when $\mathrm{Fu}$ and Kane proposed an ingenious idea to artificially engineer a topological superconductor based on a normal superconductor and the exotic properties of the electronic surface states of a topological insulator $(\mathrm{TI}){ }^{6}$ After then, a race for searching MZMs started all over the world. ${ }^{7-11}$

By superconducting proximity effect, ${ }^{12}$ superconductivity can be introduced into TI. However, because of the interface interactions, the huge lattice mismatches and the instability of Tls, the interface of a superconductor (SC) and a TI is very difficult to be established. In 2012, our group successfully fabricated the

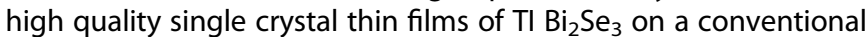
$s$-wave superconductor $\mathrm{NbSe}_{2}$ by means of molecular beam epitaxy (MBE) and demonstrated the co-existence of topological surface states (TSSs) and superconductivity in such system. ${ }^{13}$ By putting $\mathrm{TI}$ on the top of SC, this system has several significant advantages that promote the research on MZMs in this platform. First, MBE with the help of reflection high-energy electron diffraction, allows us to control the thickness of TI films as precise as one layer during the growing process. As a result, we can grow heterostructures with different thicknesses and study their superconductivity related electronic properties in detail, since the topological properties and proximity-induced superconductivity are directly affected by the TI thin film thickness. And more importantly, if the $\mathrm{TI}$ film is thin enough to make the up surface states superconducting, the resulted TSC can be studied by surface sensitive techniques directly. Second, state of the art MBE method has a reputation of growing thin films with extremely high quality. TI films with better quality compared with bulk samples will have Fermi level closer to the Dirac point. Also, Fermi level of the TI film can be tuned by doping. The Fermi level position plays a crucial role in searching MZM which will be discussed later. Third, in other systems, it is required to apply a sufficiently large magnetic field to make the system topological in the first place, in which case the bands are already spin polarized. It will then be difficult to detect the spin property of the MZM since both MZM (if present) and finite-energy midgap states would be spin polarized. In TI/SC system, a small magnetic field is sufficient to induce vortices and, hence, the MZM in the vortex core. Small magnetic field also makes this system more promising for further application.

This article mainly focuses on the recent experimental works on the $3 \mathrm{D} \mathrm{TI} / \mathrm{SC}$ heterostructure system, which is also referred as an artificial TSC. We review the structure and fabrication of the heterostructure in Section 'Topological insulator-superconductor heterostructure', and its superconducting property in Section 'Topological superconductor'. Then we discuss the detection of Majorana Zero Mode in such system in Section 'Experimental detection of Majorana zero mode' and finally give a brief summary and outlook in Section 'Summary and outlook'.

\footnotetext{
${ }^{1}$ Key Laboratory of Artificial Structures and Quantum Control (Ministry of Education), School of Physics and Astronomy, Shanghai Jiao Tong University, 200240 Shanghai, China and ${ }^{2}$ Collaborative Innovation Center of Advanced Microstructures, 210093 Nanjing, China

Correspondence: Jin-Feng Jia (jfjia@sjtu.edu.cn)
}

Received: 27 December 2016 Revised: 11 March 2017 Accepted: 31 May 2017

Published online: 03 July 2017 


\section{TOPOLOGICAL INSULATOR-SUPERCONDUCTOR HETEROSTRUCTURE}

The original proposal of a TI-SC heterostructure by Fu and Kane requires the SC on top of the $\mathrm{TI}^{6}$ However the first reported heterostructure chose an opposite scenario. The well-known 3D TI $\mathrm{Bi}_{2} \mathrm{Se}_{3}$ was grown on top of an s-wave $\mathrm{SC} \mathrm{NbSe}_{2}$ via MBE. ${ }^{14}$ Soon another similar $3 \mathrm{D} \quad \mathrm{TI} \mathrm{Bi}_{2} \mathrm{Te}_{3}$ was also grown on the same substrate. ${ }^{15,16}$ The successful fabrication of such heterostructure built a promising platform for the detection of MZMs. Here we review the material properties and growth procedures of the heterostructure and discuss some details of the heterostructure that are significant in the detection of the MZMs.

The SC substrate $2 \mathrm{H}-\mathrm{NbSe}_{2}$ is a transition-metal dichalcogenide material, a layered material that has very weak interaction between two adjacent $\mathrm{Se}-\mathrm{Nb}-\mathrm{Se}$ sheets. The in-plane lattice constant of $\mathrm{NbSe}_{2}$ is $3.44 \AA$. In bulk $\mathrm{NbSe}_{2}$, a charge density wave phase emerges below $33 \mathrm{~K}$ and a superconducting phase emerges below $7.2 \mathrm{~K}$. Bulk $\mathrm{NbSe}_{2}$ crystals are prepared via chemical vapor transport method. The size of the flakes is typically larger than two by $2 \mathrm{~mm}^{2}$, suitable as a substrate for the further MBE growth and scanning tunneling microscopy (STM) measurements.

The 3D Tls grown on top of $\mathrm{NbSe}_{2}$ are also layered materials $\mathrm{Bi}_{2} \mathrm{Se}_{3}$ and $\mathrm{Bi}_{2} \mathrm{Te}_{3}$. They have a five-atom-layer sheet structure Se (Te)-Bi-Se (Te)-Bi-Se (Te). One five-atom-layer sheet is called a quintuple layer (QL). The in-plane lattice constants of $\mathrm{Bi}_{2} \mathrm{Se}_{3}$ and $\mathrm{Bi}_{2} \mathrm{Te}_{3}$ are 4.13 and $4.38 \AA$, respectively. The most significant properties of 3D TIs are their TSSs. ${ }^{17-21}$ The TSSs lie in the bulk band gap of Tls and connect the valance band and the conduction band. Ideally, Fermi levels of the Tls lie inside the bulk band gap and cut through the TSSs only. However the Fermi levels of the grown bulk materials $\mathrm{Bi}_{2} \mathrm{Se}_{3}$ and $\mathrm{Bi}_{2} \mathrm{Te}_{3}$ always lie inside the conduction band while Fermi level of $\mathrm{Sb}_{2} \mathrm{Te}_{3}$, another 3D TI with similar structure, lies inside the valance band. This is due to the large amounts of defects inside the bulk materials.

The 3D Tls in the heterostructure are grown via MBE technique, a delicate thin film growing method that provide atomic layer thickness control ability and extreme high quality films with few defects. These two features of MBE method are crucial in the growth of $\mathrm{Tl} / \mathrm{SC}$ heterostructure:

First, as mentioned before, defects in the samples will move the Fermi level away from the Dirac points of the TSSs towards conduction band of valance band. Electrons that form superconducting gap around Fermi level will then come from the bulk bands rather than from the TSSs. However, only when superconductivity is induced into the TSSs from a SC substrate via proximity effect, Cooper pairs in the TSSs will behave "spinless" and MZMs will be hosted inside an Abrikosov vortex on the TI surface. The high quality thin films grown by MBE can overcome this problem by making the Fermi level much closer to the Dirac point.

$\mathrm{Bi}_{2} \mathrm{Se}_{3}$ and $\mathrm{Bi}_{2} \mathrm{Te}_{3}$ have been grown on several substrates. ${ }^{22-25}$ The growth conditions of the two materials are similar. The substrate temperature is controlled to be lower than the evaporation temperature of $\mathrm{Bi}$ and higher than the evaporation temperature of Se or Te for the two kinds of Tls, which is typically range from 500 to $570 \mathrm{~K}$. Under this temperature, Se or Te molecules will desorb from the substrate surface except that those form $\mathrm{Bi}_{2} \mathrm{Se}_{3}$ and $\mathrm{Bi}_{2} \mathrm{Te}_{3}$ with $\mathrm{Bi}$ atoms. In this case, the growing process can be performed under a Se (Te)-rich environment, where flux ratio of $\mathrm{Se}(\mathrm{Te})$ and $\mathrm{Bi}$ can be set to over 10. As a result, the extra amount of Se (Te) will suppress the formation of Se (Te) vacancies and antisite defects in the MBE grown films and the Fermi level of the thin films will be closer to the Dirac point.

Second, proximity effect that transfers Cooper pairs from the SC to the TI TSSs decays exponentially with the thickness of the TI film above. Once the TI films are too thick, the superconducting gap on its surface will be too small for the MZMs measurements
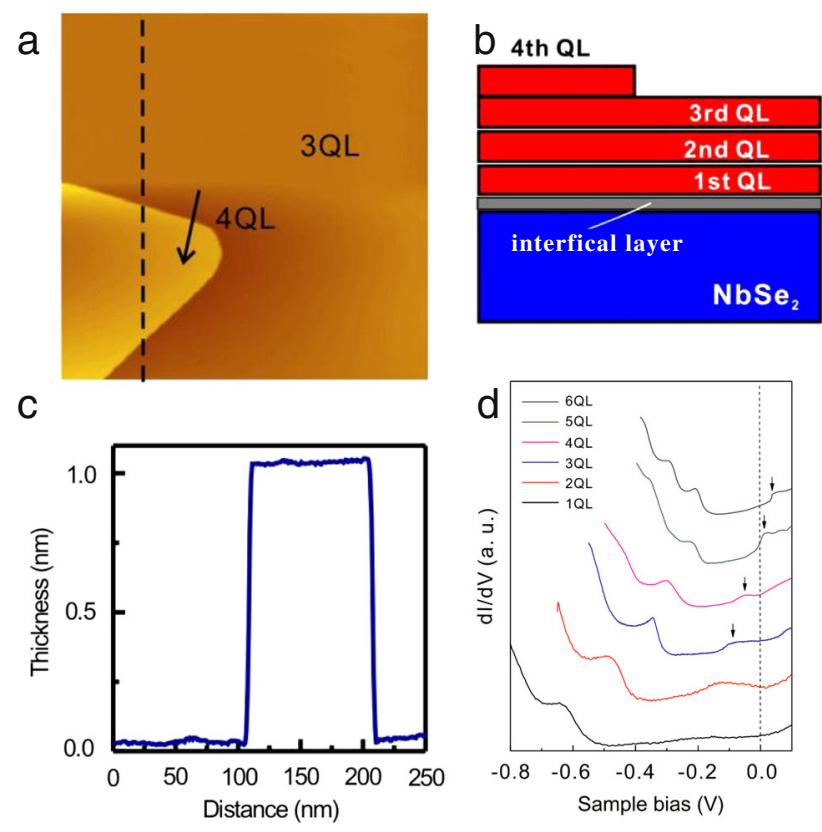

Fig. 1 a An STM image of $\mathrm{Bi}_{2} \mathrm{Te}_{3} / \mathrm{NbSe}_{2}$. b Sketch of the heterostructure. $c$ Line profile of $\mathrm{Bi}_{2} \mathrm{Te}_{3} / \mathrm{NbSe}_{2}$ at position indicated by the black dashed line in (a). d The dependence of Fermi level on the thickness of $\mathrm{Bi}_{2} \mathrm{Te}_{3}$ (reprinted with permission from ref. 16, copyright 2015 by the American Physical Society)

via STM with current energy resolution (around $0.1 \mathrm{meV}$ ), since the excitations lie exactly at zero energy inside the superconducting gap. On the other hand, TSSs of 3D TIs is fully developed after a certain layers of the materials. If the films are too thin, i.e., less than 2 QLs on Si substrate, interactions between the top and bottom surfaces will destroy the TSSs. ${ }^{23}$

$\mathrm{Bi}_{2} \mathrm{Se}_{3}$ is grown on $\mathrm{NbSe}_{2}$ at substrate temperature $500 \mathrm{~K}$. It is grown in a layer-by-layer mode. ${ }^{13}$ Bulk $\mathrm{Bi}_{2} \mathrm{Se}_{3}$ crystal has a layer thickness of $0.94 \mathrm{~nm}$ while the first layer $\mathrm{Bi}_{2} \mathrm{Se}_{3}$ on $\mathrm{NbSe}_{2}$ is as thick as $1.5 \mathrm{~nm}$. The larger thickness of the first QL suggests an interfacial layer exist between the first $\mathrm{QL} \mathrm{Bi}_{2} \mathrm{Se}_{3}$ and the $\mathrm{NbSe}_{2}$ substrate. A detailed study of this interfacial layer reveals its structure in ref. 14. An NaCl-type BiSe structure forms on the $\mathrm{NbSe}_{2}$ substrate before the formation of $\mathrm{Bi}_{2} \mathrm{Se}_{3}$ to compensate the lattice mismatch between the two.

The growth of $\mathrm{Bi}_{2} \mathrm{Te}_{3}$ on $\mathrm{NbSe}_{2}$ is very similar with $\mathrm{Bi}_{2} \mathrm{Se}_{3}$ on $\mathrm{NbSe}_{2}$. The structure of $\mathrm{Bi}_{2} \mathrm{Te}_{3}$ on $\mathrm{NbSe}_{2}$ is shown in Fig. 1. Combining MBE with RHEED technique, it is possible to control the exact QL number of the deposited thin films by monitoring RHEED oscillation pattern during the sample growth. Each intensity maximum in the RHEED oscillation pattern represents the formation of a complete $\mathrm{QL}$ of $\mathrm{Bi}_{2} \mathrm{Te}_{3}$. It is then convenient to control the thickness of the $\mathrm{Bi}_{2} \mathrm{Te}_{3}$ on top of $\mathrm{SC}$ substrate precisely to obtain a heterostructure with a measureable superconducting gap and well developed TSSs. Typically 5 QL or 6 QL samples are suitable for the detection of MZMs. At such thickness, the Fermi energy just cut through the conduction band bottom of $\mathrm{Bi}_{2} \mathrm{Te}_{3}$, as shown in Fig. 1d, which provides enough contribution of the superconductivity from the TSSs.

\section{TOPOLOGICAL SUPERCONDUCTOR}

MZMs exist in the vortex of a spinless $p_{x}+i p_{y}$ superconductor, ${ }^{26-29}$ which is called a TSC. It is proposed that via the proximity effect from an s-wave SC, the two dimensional TSSs in a TI resemble a spinless $p_{x}+i p_{y}$ like SC and thus host MZMs in vortices. ${ }^{6}$ The purpose to build a $\mathrm{TI} / \mathrm{SC}$ heterostructure is to induce superconductivity in the $\mathrm{Tl}$, or specifically in the TSSs, via proximity 

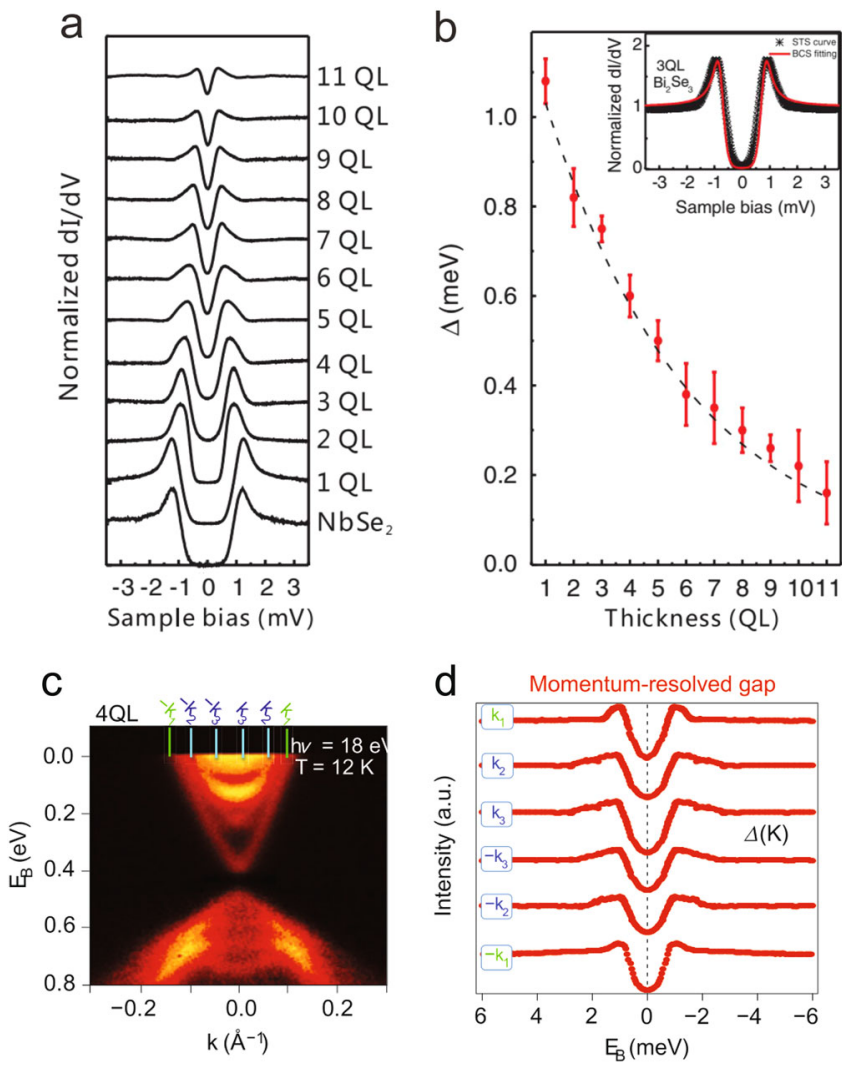

Fig. 2 a STS spectra on bare $\mathrm{NbSe}_{2}$ and $1 \mathrm{QL}$ to $11 \mathrm{QL} \mathrm{Bi}_{2} \mathrm{Te}_{3}$ surfaces, measured at $400 \mathrm{mK}$ ). b Exponential fit of the gap sizes extracted from a by the positions of the coherence peaks. c ARPES measurement of $4 \mathrm{QL} \mathrm{Bi}_{2} \mathrm{Se}_{3} / \mathrm{NbSe}_{2}$ heterostructure at $12 \mathrm{~K}$. d Symmetrized ARPES spectra at $\pm k 1, \pm k 2$, and $\pm k 3$ in $c$ at $T \sim 1 \mathrm{~K}$. a and $\mathbf{b}$ reprinted with permission from ref. 15, copyright 2014 by the American Physical Society, a and b Reprinted by permission from Macmillan Publishers Ltd: Nature Physics, ref. 30, copyright 2014

effect. In this section, we discuss the superconductivity in the TI/SC heterostructure.

Superconductivity in the TI/SC heterostructure is analyzed via both scanning tunneling spectroscopy (STS) measurements and high energy resolution angle resolved photoemission spectroscopy (ARPES) measurements, ${ }^{30}$ as shown in Fig. 2. The STS spectra clearly demonstrate gap features on the surface of the $\mathrm{TI}$ above SC. These gaps can be eliminated by raising temperature and applying magnetic field. Moreover, with increasing layer thickness of the $\mathrm{TI}$, the size of the gap decays exponentially, which is a typical behavior of the proximity induced superconductivity. Undoubtfully, the spectra gaps on the surface of the TI are superconducting gaps. Since STS provides no momentum resolution, whether TSSs contribute to the gaps is unknown. ARPES measurements explicitly answer the question. From a series of measurements at k-space of the TSSs, the density of states clearly show gaps structures near the Fermi level. The spinresolved ARPES experiment reveals that superconductivity is induced into the TSSs of the TI and two dimensional topological superconductivity in a helical TSSs is distinctly different from that in an ordinary two-dimensional superconductor in terms of the spin degrees of freedom of electrons. These results from STS and APRES demonstrate that a TI/SC heterostructure successfully creates superconductivity in the TSSs which become a spinless $p x+i p y$ like TSC.

Applying magnetic field to a type-Il superconductor will cause the emergence of Abrikosov vortices. ${ }^{31}$ As mentioned before, a spinless $p_{x}+i p_{y} \mathrm{SC}$ will host MZMs inside these vortices excited by magnetic field. Those vortices can be imaged in real space via STS mapping. Low energy bound states caused by Andreev reflection at the center of the vortices will have a high density of state inside the superconducting gap while the surroundings have zero density of state at zero temperature and a low density of state at finite temperature. Thusly, positions of the vortices are revealed by the zero bias conductance map. As shown in Fig. 3, vortices represented by purple circles form hexagonal lattices on the surface of the SC substrate, as well as the surface of the TI in the $\mathrm{TI} / \mathrm{SC}$ heterostructure. The lattice constant of the vortices, or the area owned by each vortex is determined by the magnitude of the field. Multiplying this area to the magnetic field, one should get the flux quantum $\phi_{0} \equiv e / 2 h=\vec{A} \cdot \vec{B}$, where $A$ is the area of one vortex, as shown in the red diamond in Fig. $3 a$ and e, and $B$ is the strength of the field. As can be seen from Fig. $3 b$ and $c$, decreasing the magnetic field will increase the distance between vortices, which has an interesting effect on the detection of the MZMs inside the vortices and will be discussed in the next section.

From the zero bias conductance map, we can get the coherence length of the SC in the Ginzburg-Landau theory,

$\sigma(r, 0)=\sigma_{0}+\left(1-\sigma_{0}\right) \times\{1-\tanh [r / \sqrt{2} \xi]\}$,

where $\sigma$ is the zero bias conductance, $\sigma_{0}$ is the zero bias conductance at the vortex center, $r$ is the distance from vortex center and $\xi$ is the in-plane coherence length. ${ }^{32}$ As shown in Fig. $3 \mathrm{f}$, the in-plane coherence length of the heterostructure increases with the thickness of the TI thin film above. This is in consistence with the lowering of the upper critical field of the $\mathrm{TI}$ thin film $\mathrm{H}_{\mathrm{c} 2} \sim 2.8 \mathrm{~T}$ in $3 \mathrm{QL} \mathrm{Bi}_{2} \mathrm{Te}_{3} / \mathrm{NbSe}_{2}$, compared with $\mathrm{H}_{\mathrm{c} 2}$ $\sim 3.4 \mathrm{~T}$ in $\mathrm{NbSe}_{2}$, since $H_{c 2}=\phi_{0} / 2 \pi \xi^{2}$. It is worth noting that the coherence length on the surface of the TI/SC heterostructure decreases with the increasing magnetic field, as shown in Fig. $3 \mathrm{~h}$. This phenomenon suggests that the superconductivity on the $\mathrm{TI}$ surface is not an ordinary single band s-wave superconductivity, since the coherence length of an $\mathrm{NbSe}_{2}$ remains the same under different fields. ${ }^{33,} 34$

In the $\mathrm{TI} / \mathrm{SC}$ heterostructure, superconductivity is induced into the TSSs via proximity effect. The above mentioned experimental evidence indicates that this induced superconductivity is the topological superconductivity that is different form the s-wave superconductivity of the substrate. Therefore, this heterostructure is truly a full gap artificial TSC.

\section{EXPERIMENTAL DETECTION OF MZM}

The fabrication of the artificial TSC was a big step towards the detection of the MZMs. Many theoretical and experimental works that focused on this platform gave a series of significant results on the existence of MZMs. In this section, we will first review some of the theoretical works of MZMs in the artificial TSC. Then we will discuss the experimental detection of MZMs in the artificial TSC which are strongly supported by those theories.

\section{The features of MZM predicted by theory}

In the early theoretical work, the artificial TSC was designed to have a thin layer of SC on top of $\mathrm{TI}^{6}{ }^{6}$ which is experimentally difficult to achieve. Growing TI on SC was proved to be much easier. ${ }^{14,15}$ In this artificial TSC, two well-separated MZMs exist in a vortex core on both top and bottom surface of TI thin films. ${ }^{35}$ As shown in Fig. $4 a-d$, the wave function distribution of the lowest energy bound state in the vortex evolves with the layer thickness $n$ of the TI above the SC substrate. When $n=2$, as shown in Fig. $4 \mathrm{a}$, the bound state is connected across the top and bottom surface of the TI. In Fig. $4 \mathrm{~b}$ where $n=4$, the distribution of the state on the top and bottom surface is disconnected and localized, which represents the signature of two MZMs. For larger $n$ in Fig. 4c and $d$, the coupling between the two surface layers is even weaker. 
a

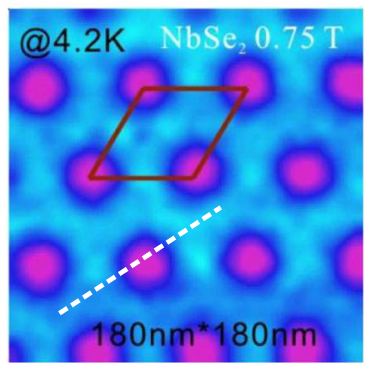

b

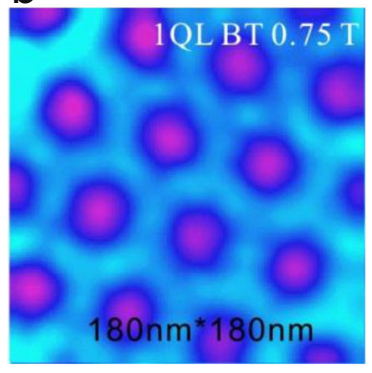

C

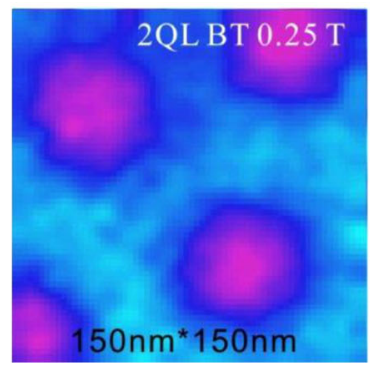

d

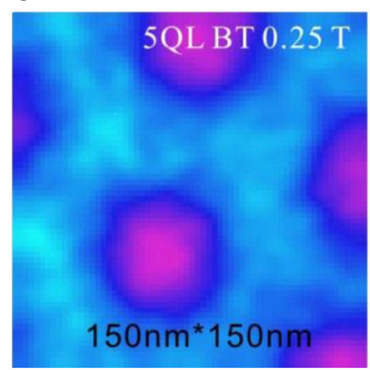

e
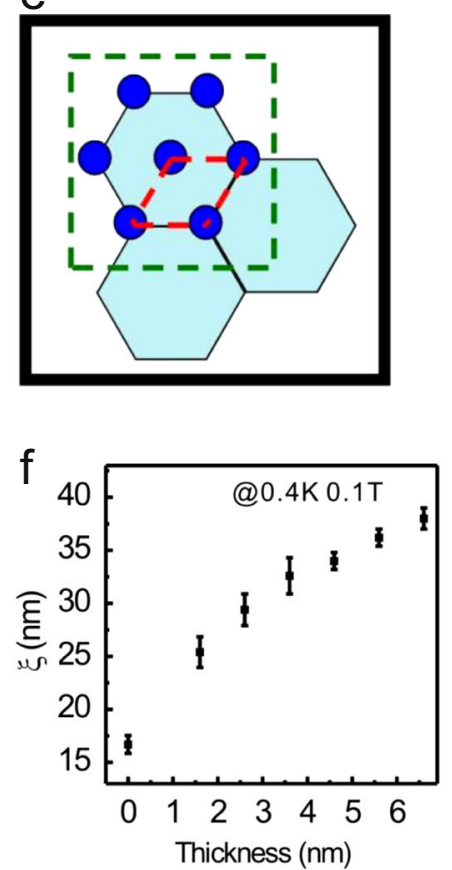

g
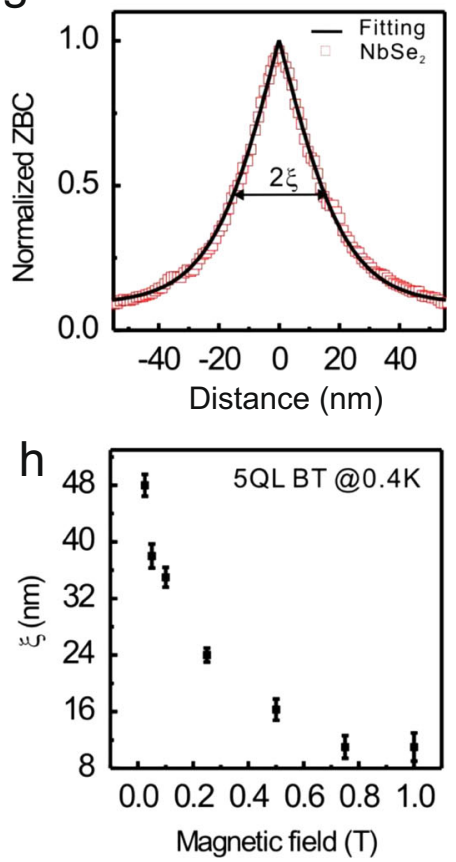

Fig. 3 Abrikosov vortices on $\mathrm{Bi}_{2} \mathrm{Te}_{3} / \mathrm{NbSe}_{2}$ heterostructure at different magnetic field. a, b $0.75 \mathrm{~T}$ for $\mathrm{NbSe}_{2}$ and $1 \mathrm{QL} \mathrm{Bi} \mathrm{Te}_{3}$. c, $\mathbf{d} 0.25 \mathrm{~T}$ for $2 \mathrm{QL}$ and $5 \mathrm{QL} \mathrm{Bi}_{2} \mathrm{Te}_{3}$. e The calculated magnetic flux of a vortex. $\mathbf{f}$ Thickness dependence of the coherence length. $\mathbf{g}$ Normalized ZBC profiles crossing through the centers of vortices at $0.4 \mathrm{~K}$ and $0.1 \mathrm{~T}$ on $\mathrm{NbSe}_{2}$. The superimposed lines are fitted results using Ginzburg-Landau theory. $\mathbf{h}$ The coherence length as a function of the magnetic field measured on $5 \mathrm{QL} \mathrm{Bi}_{2} \mathrm{Te}_{3} / \mathrm{NbSe}_{2}$. $\mathbf{f}$ and $\mathbf{h}$ reprinted with permission from ref. 15 , copyright 2014 by the American Physical Society
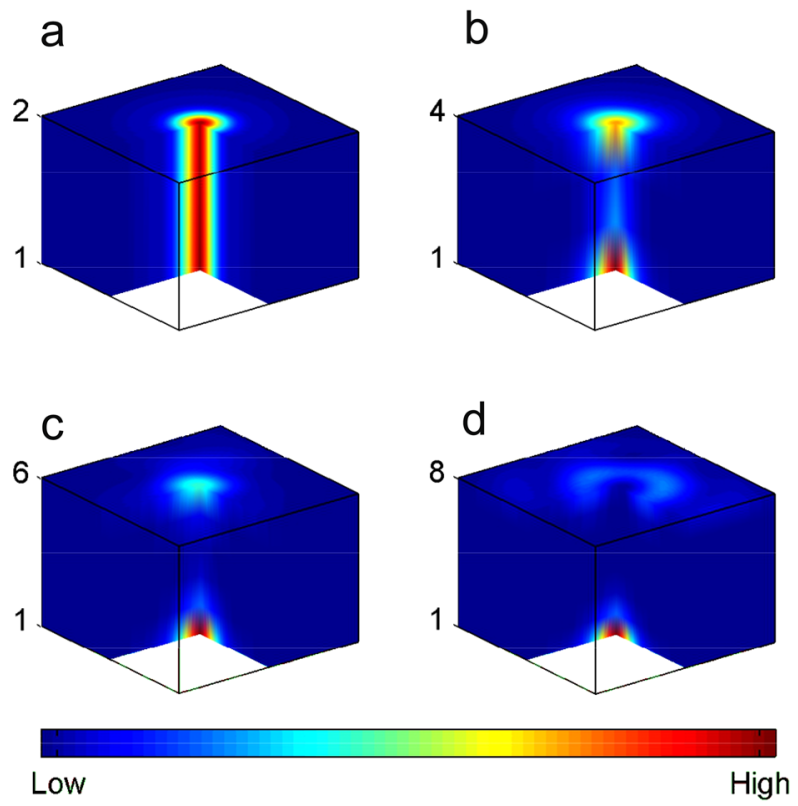

e

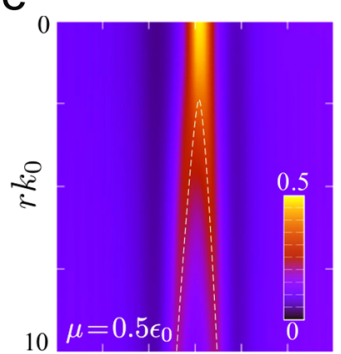

g

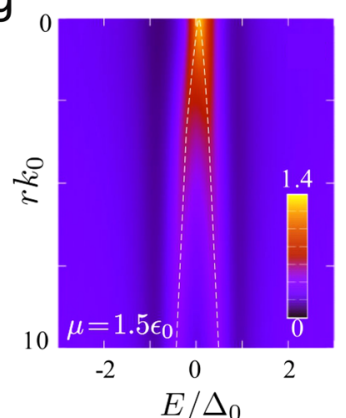

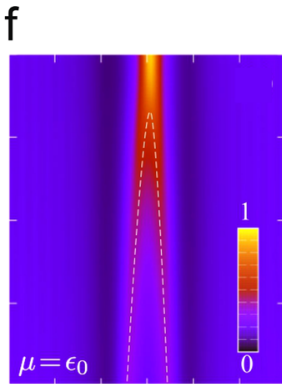

$\mathrm{h}$

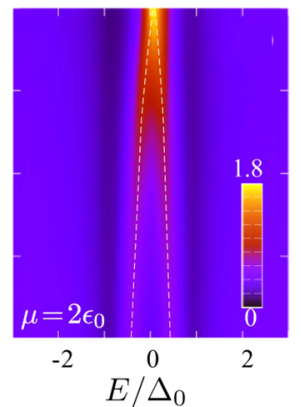

Fig. 4 Theoretical calculated Spatial distribution of MZM. a-d The wavefunction profile of MZM. a $n=2, \mathbf{b} n=4$, c $n=6$, and $\mathbf{d} n=8$. In each panel, a corner is cutoff to reveal the interior of the TI. The size of the sample is $L \times L$, where $L=15 \xi_{0}$ and $\xi_{0}$ is the coherence length of the superconducting substrate. $\mathbf{e}-\mathbf{h}$ The local densitity of states as a function of vortex radius $r$ and STS measurement energy $E$. Dotted curves are guides for the eye. $2 \varepsilon_{0}$ is the size of the band gap of the TI film, $\mu$ is the distance between the Fermi level and the dirac point of the TI. $\Delta_{0}$ is the proximity induced superconducting gap. $k_{0}^{-1}=\nu_{F} / \varepsilon_{0} \approx 2 \mathrm{~nm}$, where $v_{F}$ is the Fermi velocity of TI's surface state. a-d reprinted by permission from Macmillan Publishers Ltd: Scientific Reports, ref. 35, copyright 2014. e-h reprinted with permission from ref. 42 , copyright 2015 by the American Physical Society 
As a result, two MZMs become more disconnected in the $z$ direction and more extended in the surfaces. It is worth noting that as the MZMs get wider, they become more fragile. Another effect caused by the increase of Tl's thickness is the decay of the pairing gap induced via proximity effect. It is therefore important to control the Tl's thickness of the heterostructure in order to get fully developed, as well as robust MZMs in the vortices of the surface.

Since STM has both real space and energy space resolution, it is the best tool to detect such a MZM inside a vortex core on the TI's top surface. However there exist other low energy quasiparticle states in the vortex core that separated from the MZM by tiny energy gap, i.e., the Caroli-de Gennes-Matricon states. ${ }^{36-39}$ The

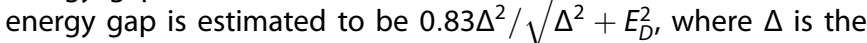
superconducting gap and $E_{D}$ is the Fermi energy relative to the Dirac point of the TSS band. ${ }^{40}$ In this TI/SC system, the typical energy gap size is $\Delta \sim 1 \mathrm{meV}$ and the Fermi level is $E_{D} \sim 100 \mathrm{meV}$, which leads to the minigap with size $\sim 0.01 \mathrm{meV}$. This is much smaller than the present energy resolution $(0.1 \mathrm{meV})$ in STS. There are two ways to increase the mini gap. One is to tune the Fermi level toward the Dirac point. However, in that case, the superconducting gap $\Delta$ becomes very small and the transition temperature becomes very low due to the weaker proximity effect. The other is using another SC material with larger gap, which is yet to be found. Hence, the direct observation of the Majorana mode is still difficult.

Fortunately, the MZM is not a point at the center of a vortex core, but extensively distributes around the core center, ${ }^{41}$ which gives an opportunity to detect the MZM by investigating the spatial distribution of the bound states in the vortex core. This spatial distribution of MZM is different from other low energy bound states inside the vortex. Shifting the Fermi energy level from TSSs towards the bulk bands, the spatial distribution of the core state will change from a Y-shape when MZM exists to a Vshape when only other low energy excitation states exist in STS measurements with finite resolution ${ }^{42}$ (see Fig. 4e-h).

Moreover, the MZM inside a vortex undergoes unique spinselective Andreev Reflection (SSAR) process. This process was first proposed in a semiconductor wire/SC structure in which MZMs exist at two ends of the wire. ${ }^{43}$ The proposed SSAR of the MZM in 1D wire can be generalized to a 2D TSC. In the latter case, the spin of the MZM in the vortex core has a spatial distribution and is not conserved. However, the spin wave function at the center of the MZM at the vortex core is fully polarized along the external magnetic field. Therefore, the Andreev reflection at the vortex center is expected to be spin-selective. ${ }^{42-44}$ Via an spin-polarized $\mathrm{STM}^{45}{ }^{46}$ we can inject spin-polarized tunneling current in STS measurements and observe the zero bias conductance change caused by the SSAR from the MZM when flipping the spin of the tunneling electrons. ${ }^{47,} 48$ It is worth noting that only a small magnetic field is sufficient to excite vortices on the TI surface and hence the spin-polarized MZM in the vortex core. On the other hand, the spin polarization of other surface states and finite energy quasiparticle states is still negligibly small. This is a unique advantage to probe the SSAR in the TSC. In other systems, i.e., in Rashba wire systems, a sufficiently large magnetic field is required to lift the spin degeneracy and make the system topological in the first place, in which case the bands are already spin polarized. It will then be difficult to attribute spin-dependent zero-bias conductance to the MZM since both MZM (if present) and finiteenergy midgap states would be spin polarized.

\section{Zero bias peak}

As mentioned above, energy resolution in most STS measurements is not sufficient to distinguish the MZM from other low energy bound states in the vortex core. All these excitations will merge together and form a single peak centered at zero energy with certain broadening width in an STS measurement. This peak is referred as the zero bias peak (ZBP). Though we cannot separate the MZM from other excitations, the conductance contributed from the MZM at zero energy reveals MZM's existence. In the artificial TSC formed by $\mathrm{Bi}_{2} \mathrm{Te}_{3} / \mathrm{NbSe}_{2}$ heterostructure, a series of STS measurements focused on the ZBP at the center of the vortex on the TI's surface were performed and the results are given in Fig. 5. STS spectra taken at the vortex core under magnetic fields ranging from $0.025 \mathrm{~T}$ to $1.00 \mathrm{~T}$ on three different samples, $5 \mathrm{QL}$ $\mathrm{Bi}_{2} \mathrm{Te}_{3} / \mathrm{NbSe}_{2}, 2 \mathrm{QL} \mathrm{Bi}_{2} \mathrm{Te}_{3} / \mathrm{NbSe}_{2}$ and bare $\mathrm{NbSe}_{2}$, are shown in Fig. 5a-c respectively. In Fig. $5 \mathrm{a}$, the spectra taken on $5 \mathrm{QL} \mathrm{Bi}_{2} \mathrm{Te}_{3} /$ $\mathrm{NbSe}_{2}$ under magnetic field ranging from $0.25 \mathrm{~T}$ to $0.10 \mathrm{~T}$ are obviously different from others. The intensities of the ZBP in these spectra are dramatically higher than the others. In Fig. 5b, such ZBP intensity anomaly is absent on the $2 \mathrm{QL} \mathrm{Bi}_{2} \mathrm{Te}_{3} / \mathrm{NbSe}_{2}$. In Fig. $5 c$, the ZBP intensity anomaly is again absent on the $\mathrm{NbSe}_{2}$
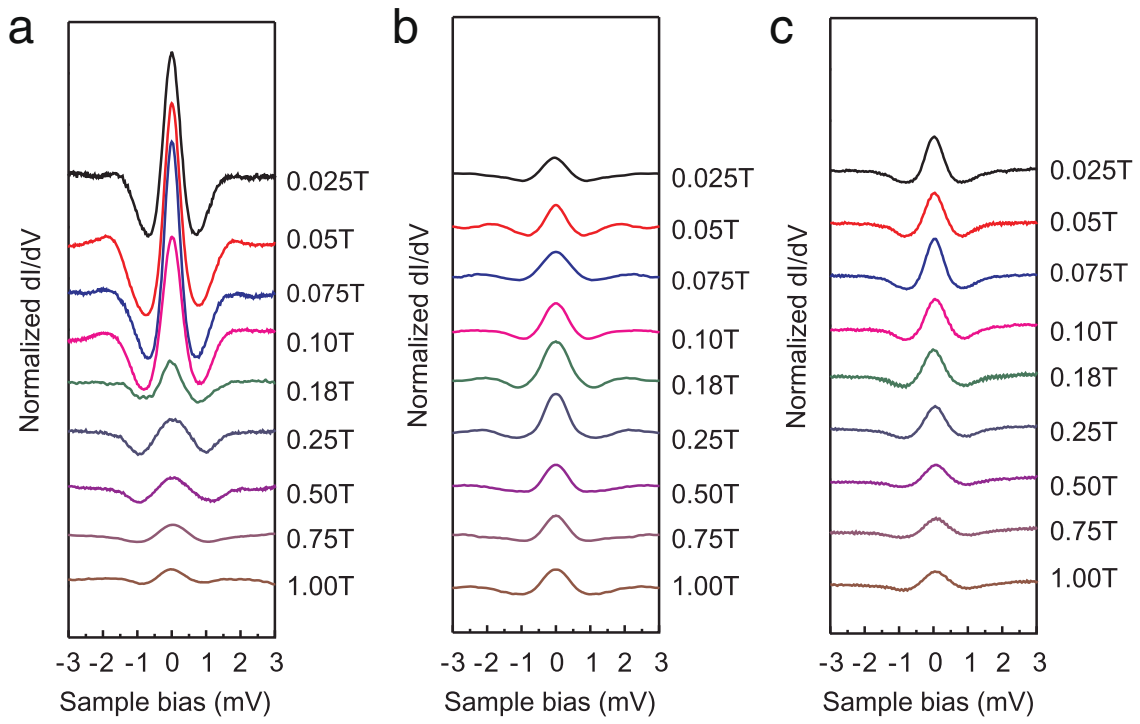

Fig. 5 A series of STS spectra measured at various magnetic fields at a vortex center of $5 \mathrm{QL} \mathrm{Bi}_{2} \mathrm{Te}_{3} / \mathrm{NbSe}_{2} \mathbf{a}, \mathrm{bare} \mathrm{NbSe}_{2}, \mathbf{b}$ and 2 QL Bi $2 \mathrm{Te}_{3} /$ $\mathrm{NbSe}_{2}$ c. A dramatic drop in the peak intensity is clearly seen at $0.18 \mathrm{~T}$ in (a). a-c reprinted with permission from ref. 16 , copyright 2015 by the American Physical Society 
a
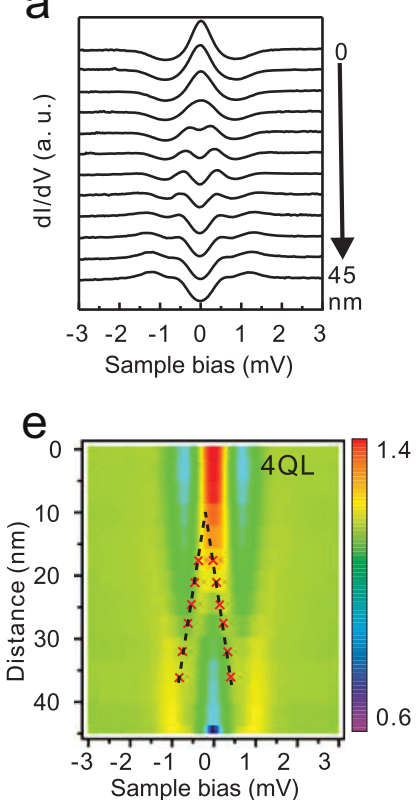

b

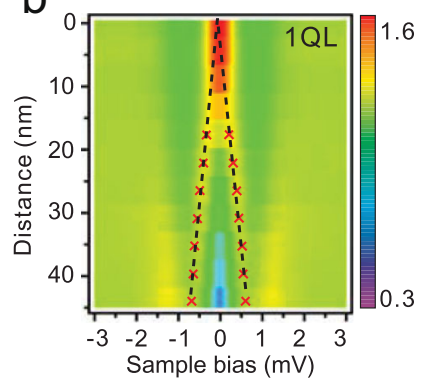

f

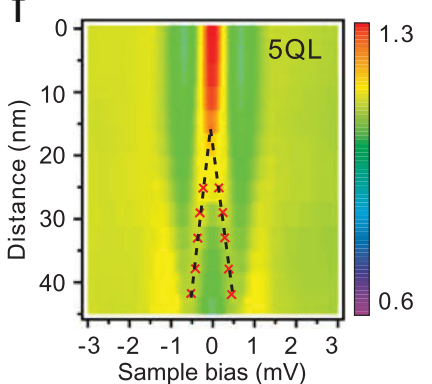

C

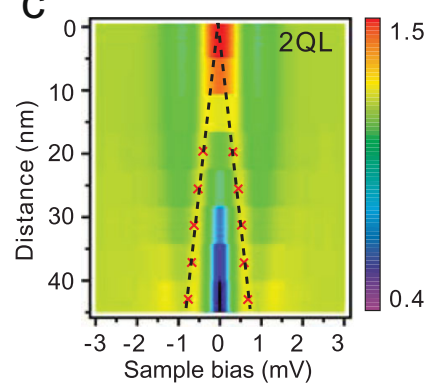

g

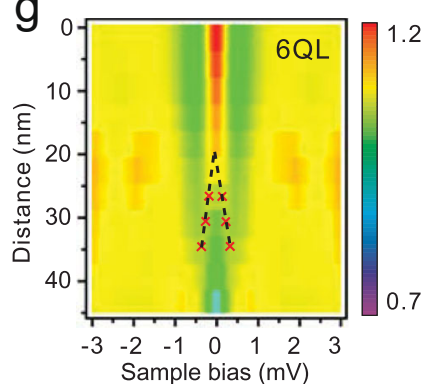

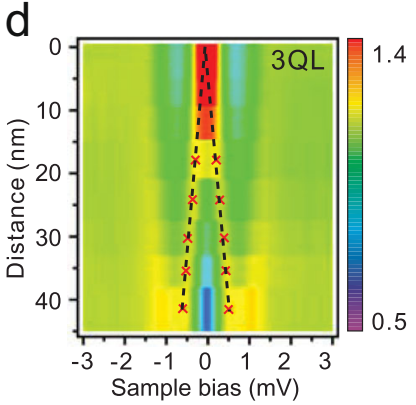

$\mathrm{h}$

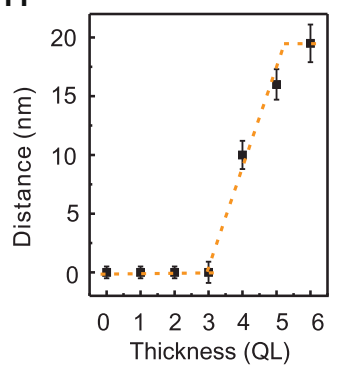

Fig. 6 a A series of STS spectra curves measured along the line starting from the core to the edge of the vortex, showing the peak of bound states splits into two at positions away from the vortex center. $\mathbf{b}$ The color image of a for a better view. The split peak positions in the STS spectra are marked by red crosses, and the dotted lines superimposed on the crosses indicate the start point of the peak splitting. c- $\mathbf{g}$ The experimental results for 2-6 QL samples, following the similar data process of (b). $\mathbf{h}$ A summary of the start points of the peak split, showing a crossover at 4 QL. a-h reprinted with permission from ref. 16, copyright 2015 by the American Physical Society)

substrate. This fact shows the ZBP intensity anomaly cannot be observed on normal s-wave superconductors. This ZBP anomalous behavior is therefore believed to come from the contribution of the MZM in the vortex core of the $5 \mathrm{QL} \mathrm{Bi}_{2} \mathrm{Te}_{3} / \mathrm{NbSe}_{2}$ sample. In the $2 \mathrm{QL} \mathrm{Bi}_{2} \mathrm{Te}_{3} / \mathrm{NbSe}_{2}$, the Fermi level lies deep in the bulk bands of the TI and does not cut through the TSSs (see Fig. 1). Thusly, the bulk carriers dominate in the ZBPs, so they behavior just like those in s-wave superconductor. In this sample, the absence of the ZBP intensity anomaly also shows that the anomaly is not due to the proximity effect. In Fig. 5a, the ZBP anomaly only shows up at magnetic field lower than $0.18 \mathrm{~T}$. At higher field, the intensities of the ZBPs are comparable to those in 2 QL samples. This phenomenon can be explained by the distance between two adjacent vortices and the spatial distribution of MZM's wavefunction. As mentioned before, two adjacent vortices will come closer when the excitation magnetic field is increased (see Fig. 3). Meanwhile, MZM has a spatial distribution centered at vortex core (see Fig. 4a-d). The MZM spatial distribution is around $100 \mathrm{~nm}$, which is close to the distance between two adjacent vortex at $0.18 \mathrm{~T}$. When the field is large enough, the two MZMs at two adjacent vortices cores overlap and annihilate each other. ${ }^{16}$ More evidence of this argument is discussed in the following experiment that focus of the spatial distribution of MZMs (see Figs. 6 and 7) and the SSAR effect (see ref. 48).

\section{Spatial distribution}

Besides from the ZBP anomaly, the spatial distribution of MZMs in the artificial TSC were also detected experimentally. The spatial distribution of a MZM has a wide extension centered at vortex core, with its energy being exactly at zero. The spatial distribution of other low energy quasiparticle excitations have energy dispersions correlated between their spatial positions of the maximal absolute value of their wave functions. As a general rule, a quasiparticle with higher energy locates at a position further away from the center of vortex (see Fig. $6 \mathrm{a}$ ). In the $\mathrm{Bi}_{2} \mathrm{Te}_{3} / \mathrm{NbSe}_{2}$ heterostructure, STS spectra were taken along a line starting from the center of the vortex to the edge of the vortex on samples with various layer thickness of $\mathrm{TI}$ above SC. Two kinds of spatial distributions of the quasiparticle excitations in the vortex are observed via STS, ${ }^{16}$ as shown in Fig. 6 . In samples from 1 QL to 3 QL layers of $\mathrm{Tl}$, the splitting of the excitations starts from the vortex core, and extend linearly towards the vortex edge, forming a V-shape pattern (see Fig. $6 \mathrm{~b}-\mathrm{d}$ ). In samples from $4 \mathrm{QL}$ to $6 \mathrm{QL}$ layers of $\mathrm{Tl}$, the splitting starting point shifts away from the vortex center, forming a Y-shape pattern. With increasing layer thickness, the splitting point is further away. The Y-shape splitting pattern originates from the MZM at the center of the vortex. The wide spatial distribution of MZM smears the splitting of other low energy excitations and causes a large ZBP around the vortex core. As shown in Fig. $4 a-d$, the spatial distribution of the MZM become wider when the TI become thicker. This explains the why splitting points shifts outside with the increasing TI layer thickness.

More importantly, we found that when the ZBP anomaly appears, the Y-shape splitting pattern appears, and they also disappear together. As shown in Fig. 7a, b, d and e, the Y-shape splitting pattern can never be observed on s-wave superconductor $\mathrm{NbSe}_{2}$, or $1 \mathrm{QL} \mathrm{Bi} \mathrm{Te}_{3} / \mathrm{NbSe}_{2}$. It can only be observed on thick TI films, like $5 \mathrm{QL} \mathrm{Bi} \mathrm{Te}_{3} / \mathrm{NbSe}_{2}$ and at low magnetic field (below 0.18 $\mathrm{T}$, Fig. 7c). When the magnetic field is larger than $0.18 \mathrm{~T}$, the $\mathrm{Y}$ shape splitting pattern chances back to the V-shape splitting pattern (Fig. 7f). The difference between low field and heigh field which only exists in the sample with MZMs strongly suggests that this difference is caused by the destroying of the MZMs field due to the interaction between them when the field gets larger and the vortices get closer.

If we cut along zero bias in Fig. $7 c$ and $f$, two curves are obtained. The one from Fig. 7c contains the contribution from $M Z M$, and the one from Fig. $7 f$ does not. So, we can extract the contribution of MZM alone by subtracting them. Those curves and their subtracted result are shown in Fig. $7 \mathrm{~g}$, with rotation along $z-$ axis, from which the spatial distribution of a MZM can be deduced. The cone-like shape agrees quite well with the calculated one (Fig. 7h) from ref. 41. 

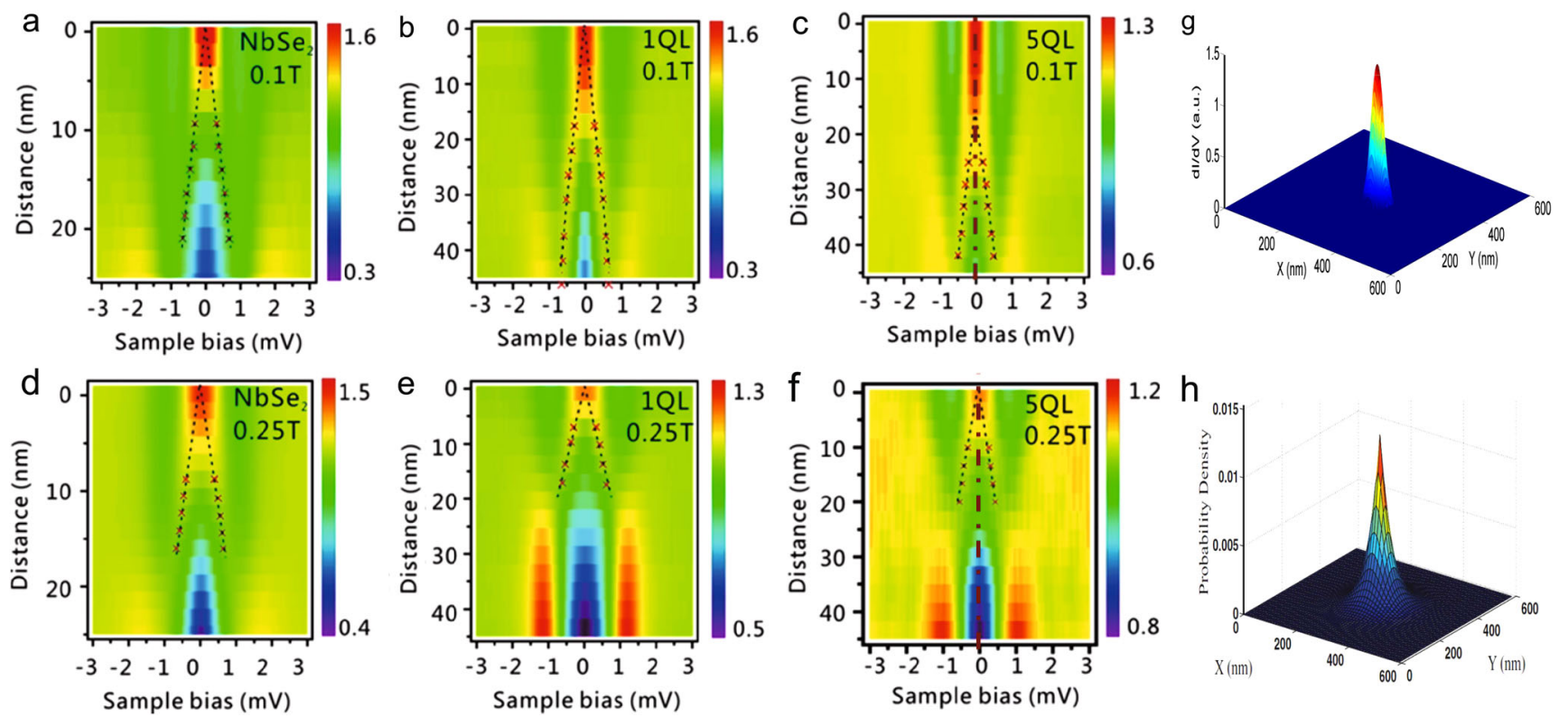

Fig. 7 a-f Comparison of the splitting starting point for different samples and at different magnetic fields. The $Y$-shape splitting pattern can only be observed on $5 \mathrm{QL} \mathrm{Bi}_{2} \mathrm{Te}_{3}$ sample at $0.1 \mathrm{~T}$ magnetic field. $\mathbf{g}$ Spatial distribution of the $\mathrm{MZM}$ detected in the vortex core on a 5 QL Bi $\mathrm{Te}_{3} /$ $\mathrm{NbSe}_{2}$ sample. $\mathbf{h}$ The spatial distribution of MZM in the vortex calculated in ref. 41 (reprinted with permission from ref. 41 , copyright 2011 by the American Physical Society)

\section{Spin-selective Andreev reflection}

Via a spin-polarized STM, we can observe the SSAR on the MZM in the artificial TSC. When an electron with the same spin as the MZM's inside is injected into the vortex core, SSAR will cause the reflection of a hole with the same spin. On the other hand, when the injected electron has the spin opposite to the MZM's spin, the SSAR will cause the reflection of the electron directly. The difference of the reflected particles in the SSAR process leads to a difference in the tunneling conductance in the STS. Injecting an electron and reflecting a hole will give a high tunneling conductance while injecting an electron and reflecting an electron as well will give a low tunneling conductance. The total tunneling conductance during the process consists of two parts,

$\frac{d l(r, E ; \hat{B}, \hat{M})}{d V}=\left.\frac{d l(r, E ; \hat{B}, \hat{M})}{d V}\right|_{n}+\left.\frac{d l(r, E ; \hat{B}, \hat{M})}{d V}\right|_{A}$,

where the first term is the contribution from the normal tunneling, and the second term from the SSAR. $E$ is the energy, $r$ is the distance from the vortex core, $\hat{B}$ and $\hat{M}$ are the orientations of the external magnetic field $B$ and spin polarization $M$, respectively. It is worth noting that the SSAR is most significant at the vortex center, where the superconducting order parameter vanishes and the SSAR is purely contributed from the MZM. The term $d I /\left.d V\right|_{n}$ is proportional to the local density of states caused by normal tunneling, which is independent of spin polarization at $r=0$ and $E$ $=0$ within the energy resolution of about $0.1 \mathrm{meV}$. Therefore the difference of spin-dependent conductance probes the SSAR at the vortex core.

In the experiment, the direction of the ferromagnetic Fe-coated W tip's polarization and the direction of the magnetic field that excited the vortex and the MZM within are controlled to be both parallel and antiparallel to each other. The STS measurements are conducted in all four tip-polarization and magnetic field configurations. In the STS measurements on a $5 \mathrm{QL} \mathrm{Bi}_{2} \mathrm{Te}_{3} / \mathrm{NbSe}_{2}$, the ZBP at vortex core clearly shows a conductance difference between the parallel and antiparallel situations, as shown in Fig. $8 \mathrm{~b}$. This result demonstrates the SSAR process caused by a spin polarized tunneling current reflected by the MZM. It is worth noting that this SSAR process can only happen at the center of the vortex. When probed at a distance from the vortex core, no such effect can be seen, as shown in Fig. 8c. The experimental results fit quite well with the theoretical calculations (See Fig. $8 \mathrm{~d}$ and e).

The SSAR effect was also carefully examined in some other TI/SC samples without $\mathrm{MZM}$, i.e., $3 \mathrm{QL} \mathrm{Bi}_{2} \mathrm{Te}_{3} / \mathrm{NbSe}_{2}$ sample and bare $\mathrm{NbSe}_{2}$ substrate. In those samples, the conductance in the ZBP of two different tip polarization-magnetic field direction configurations (parallel and antiparallel) exhibit no obvious difference. As mentioned before, the absence of the SSAR rules out MZM's origin from the proximity effect or from the substrate. Similarly, in the 5 $\mathrm{QL} \mathrm{Bi}_{2} \mathrm{Te}_{3} / \mathrm{NbSe}_{2}$ sample, when the excitation field is increased to a point where two adjacent vortices is too close to each other, the MZMs are destroyed and the SSAR effect disappears accordingly. Therefore, all evidences consistently prove that the MZMs exist in the vortex.

\section{SUMMARY AND OUTLOOK}

Initially proposed by Fu and Kane in 2008, the artificial TSC system made of the $3 \mathrm{D} \mathrm{TI} / \mathrm{SC}$ heterostructure inspired vast fascinating theoretical works and significant experimental discovery in less than a decade. MZM has been proven to exist in TSC. Though a $p$ wave TSC is yet out-of-reach in nature, topological superconductivity can be achieved by inducing superconductivity into the TSSs of TIs from normal s-wave SCs. The combination of state-of-the-art MBE and STM/STS technique plays a crucial role in the discovery of MZMs. Extreme high quality $\mathrm{TI} / \mathrm{SC}$ heterostructures can be fabricated and transferred to an STM immediately for in-situ measurements. Low temperature condition (300 to $30 \mathrm{mK}$ ) and vector magnetic field help to observe the genuine SSAR properties of the mysterious MZMs. Although the detection of a single MZM lies in the minigap of other low energy quasiparticle states is still beyond current STS energy resolution, the nice agreements between theories and experimental observations and the selfconsistency in different independent experiments explicitly prove the existence of MZM in the artificial TSC.

The MZM in solid-state physics attracts great attention not only because it is a new quasiparticle in the diverse family formed by different topology phases of matter, it has serious applications in quantum computation as well. Its topological protected 

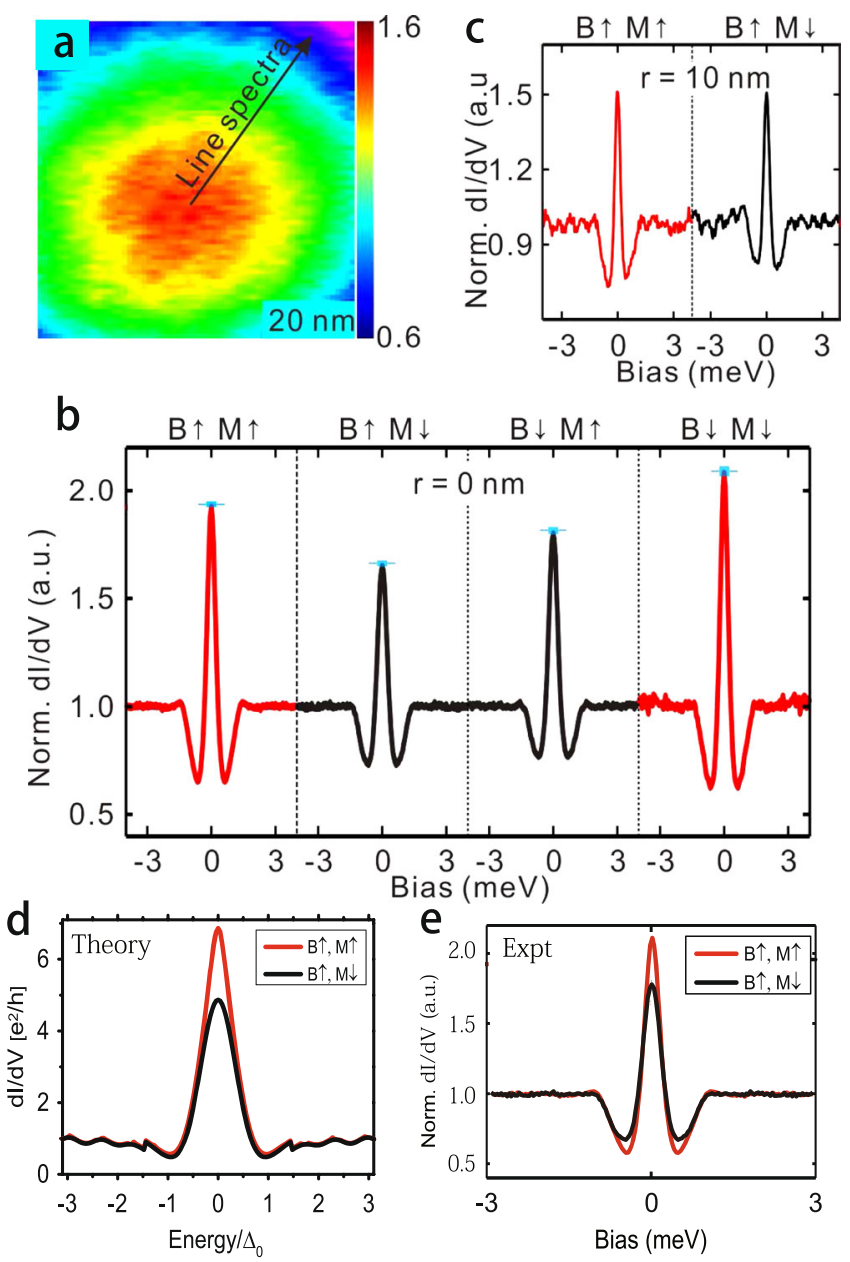

Fig. 8 a Zero bias $\mathrm{dl} / \mathrm{dV}$ mapping of a vortex at $0.1 \mathrm{~T}$ with the spin non-polarized tip on the topological superconductor $5 \mathrm{QL} \mathrm{Bi}_{2} \mathrm{Te}_{3} /$ $\mathrm{NbSe}_{2}$. b dl/dV at the vortex center measured with a fully spin polarized tip. Red curves are for parallel configuration and black curves are for antiparallel configuration. The blue lateral lines give the average values of the intensities in multi-measurements, the vertical bars are the standard error bars. c dl/dV at $10 \mathrm{~nm}$ away from the center of a vortex measured with a fully spin polarized tip, where the tunneling is found independent of the spin polarization. $\mathbf{d}$ and $\mathbf{e}$ Theoretical calculation of the $\mathrm{dl} / \mathrm{dV}$ curves in two configurations and the experimental results accordingly. a-e reprinted with permission from ref. 48, copyright 2016 by the American Physical Society

wavefunction can be used as qubits to proceed fault-tolerant quantum computation. 5,27 The non-Abelian braiding of MZMs that is needed in the fault-tolerant quantum computation is a challenging task. Recently, another approach called surface code provides an alternative way to apply MZMs in quantum computation. ${ }^{49-51}$ This method does not require the non-Abelian statistics of MZMs, so it is not a fault-tolerant quantum computation. However, it still has remarkable ability for error detection and subsequent correction during qubit readout, as the nucleation of anyons through the action of a random operator can be reliably tracked through stabilizer measurements and it is much easier to achieve experimentally. ${ }^{50}$

To realize the theoretical proposal that applies MZMs into quantum computation, much effort is needed based on the current 3D TI/SC platform. First, it requires novel fabrication methods to build a TI/SC heterostructure with fine structures to form a device that can be used to measure the interactions between multiple MZMs. Second, a robust way of manipulating
MZMs is needed to perform the necessary gate operations for universal quantum computation. Third, it is important to explore new materials that host MZMs, i.e., a better SC substrate that has larger gap protecting the MZMs. Considering the fast development in the first decade in this field, these tasks will hopefully be accomplished in the near future.

\section{ACKNOWLEDGEMENTS}

We thank the Ministry of Science and Technology of China (Grants No. 2016YFA0301003, No. 2013CB921902, No. 2014CB921201, No. 2014CB921203, No. 2014CB921103), NSFC (Grants No. 11634009, No. 11521404, No. 11504230 No. 11574202 , No. 11574201) for partial support.

\section{AUTHOR CONTRIBUTION}

H. H. S. and J. F. J. wrote the manuscript and prepared the figures.

\section{ADDITIONAL INFORMATION}

Competing interests: The authors declare that they have no competing financial interests.

Publisher's note: Springer Nature remains neutral with regard to jurisdictional claims in published maps and institutional affiliations.

\section{REFERENCES}

1. Majorana, E. Teoria simmetrica dell'elettrone e del positrone. Nuovo. Cim 14, 171-184 (1937).

2. Wilczek, F. Majorana returns. Nat. Phys 5, 614-618 (2009).

3. Elliott, S. R. \& Franz, M. Colloquium: Majorana fermions in nuclear, particle, and solid-state physics. Rev. Mod. Phys. 87, 137-163 (2015).

4. Avignone, F. T., Elliott, S. R. \& Engel, J. Double beta decay, Majorana neutrinos, and neutrino mass. Rev. Mod. Phys. 80, 481-516 (2008).

5. Nayak, C., Simon, S. H., Stern, A., Freedman, M. \& Das Sarma, S. Non-Abelian anyons and topological quantum computation. Rev. Mod. Phys. 80, 1083-1159 (2008).

6. Fu, L. \& Kane, C. L. Superconducting proximity effect and Majorana fermions at the surface of a topological insulator. Phys. Rev. Lett. 100, 096407 (2008).

7. Das, A. et al. Zero-bias peaks and splitting in an Al-InAs nanowire topological superconductor as a signature of Majorana fermions. Nat. Phys 8, 887-895 (2012).

8. Deng, M. T. et al. Anomalous zero-bias conductance peak in a Nb-InSb Nanowire-Nb hybrid device. Nano. Lett. 12, 6414-6419 (2012).

9. Mourik, V. et al. Signatures of Majorana fermions in hybrid superconductorsemiconductor nanowire devices. Science 336, 1003-1007 (2012).

10. Finck, A. D. K., Van Harlingen, D. J., Mohseni, P. K., Jung, K. \& Li, X. Anomalous modulation of a zero-bias peak in a hybrid nanowire-superconductor device. Phys. Rev. Lett. 110, 126406 (2013).

11. Nadj-Perge, S. et al. Observation of Majorana fermions in ferromagnetic atomic chains on a superconductor. Science 346, 602-607 (2014).

12. McMillan, W. L. Tunneling model of the superconducting proximity effect. Phys. Rev 175, 537-542 (1968).

13. Wang, M.-X. et al. The coexistence of superconductivity and topological order in the Bi2Se3 thin films. Science 336, 52-55 (2012).

14. Mei-Xiao, W. et al. Interface structure of a topological insulator/superconductor heterostructure. New J. Phys. 16, 123043 (2014).

15. Xu, J.-P. et al. Artificial topological superconductor by the proximity effect. Phys. Rev. Lett. 112, 217001 (2014).

16. $\mathrm{Xu}, \mathrm{J}$.-P. et al. Experimental detection of a majorana mode in the core of a magnetic vortex inside a topological insulator-superconductor $\mathrm{Bi}_{2} \mathrm{Te}_{3} / \mathrm{NbSe}_{2}$ heterostructure. Phys. Rev. Lett. 114, 017001 (2015).

17. Xia, Y. et al. Observation of a large-gap topological-insulator class with a single Dirac cone on the surface. Nat. Phys 5, 398-402 (2009).

18. Zhang, $\mathrm{H}$. et al. Topological insulators in $\mathrm{Bi} 2 \mathrm{Se} 3, \mathrm{Bi} 2 \mathrm{Te} 3$ and $\mathrm{Sb} 2 \mathrm{Te} 3$ with a single Dirac cone on the surface. Nat. Phys 5, 438-442 (2009).

19. Chen, Y. L. et al. Experimental realization of a three-dimensional topological insulator Bi2Te3. Science 325, 178-181 (2009).

20. Hasan, M. Z. \& Kane, C. L. Colloquium: topological insulators. Rev. Mod. Phys. 82, 3045-3067 (2010).

21. Qi, X.-L. \& Zhang, S.-C. Topological insulators and superconductors. Rev. Mod. Phys. 83, 1057-1110 (2011). 
22. Song, C.-L. et al. Topological insulator Bi2Se3 thin films grown on double-layer graphene by molecular beam epitaxy. Appl. Phys. Lett. 97, 143118 (2010).

23. $\mathrm{Li}, \mathrm{Y}$.-Y. et al. Intrinsic topological insulator $\mathrm{Bi} 2 \mathrm{Te} 3$ thin films on $\mathrm{Si}$ and their thickness limit. Adv. Mater. 22, 4002-4007 (2010).

24. He, L. et al. Epitaxial growth of Bi2Se3 topological insulator thin films on $\mathrm{Si}$ (111). J. Appl. Phys. 109, 103702 (2011).

25. Liu, X. et al. Characterization of $\mathrm{Bi} 2 \mathrm{Te} 3$ and $\mathrm{Bi} 2 \mathrm{Se} 3$ topological insulators grown by MBE on (001) GaAs substrates. J. Vac. Sci. Technol. B 30, $02 B 103$ (2012).

26. Read, N. \& Green, D. Paired states of fermions in two dimensions with breaking of parity and time-reversal symmetries and the fractional quantum Hall effect. Phys. Rev. B 61, 10267-10297 (2000).

27. Ivanov, D. A. Non-Abelian statistics of half-quantum vortices in p-wave superconductors. Phys. Rev. Lett. 86, 268-271 (2001).

28. Stern, A., von Oppen, F. \& Mariani, E. Geometric phases and quantum entanglement as building blocks for non-Abelian quasiparticle statistics. Phys. Rev. $B$ 70, 3352-3359 (2004).

29. Stone, M. \& Chung, S.-B. Fusion rules and vortices inpx + ipysuperconductors. Phys. Rev. B 73, 014505 (2006).

30. $\mathrm{Xu}, \mathrm{S} .-\mathrm{Y}$. et al. Momentum-space imaging of cooper pairing in a half-dirac-gas topological superconductor. Nat. Phys 10, 943-950 (2014).

31. Abrikosov, A. A. Magnetic properties of superconductors of the second group. Sov. Phys.-JETP 5, 1174-1183 (1957).

32. Eskildsen, M. R. et al. Vortex imaging in the pi band of magnesium diboride. Phys. Rev. Lett. 89, 187003 (2002)

33. Nakai, N. et al. Ubiquitous V-shape density of states in a mixed state of clean limit type II superconductors. Phys. Rev. Lett. 97, 147001 (2006).

34. Fente, A. et al. Field dependence of the vortex core size probed by scanning tunneling microscopy. Phys. Rev. B 94, 014517 (2016).

35. Li, Z.-Z., Zhang, F.-C. \& Wang, Q.-H. Majorana modes in a topological insulator/swave superconductor heterostructure. Sci. Rep 4, 6363 (2014).

36. Caroli, C., De Gennes, P. G. \& Matricon, J. Bound Fermion states on a vortex line in a type II superconductor. Phys. Lett. 9, 307-309 (1964).

37. Mints, R. G. \& Rachmanov, A. L. On the energy spectrum of excitations in type-II superconductors. Solid State Commun 16, 747-750 (1975).

38. Kraus, Y. E., Auerbach, A., Fertig, H. A. \& Simon, S. H. Testing for majorana zero modes in a $\mathrm{p}(\mathrm{x})+\mathrm{ip}(\mathrm{y})$ superconductor at high temperature by tunneling spectroscopy. Phys. Rev. Lett. 101, 267002 (2008).

39. Akzyanov, R. S., Rozhkov, A. V., Rakhmanov, A. L. \& Nori, F. Tunneling spectrum of a pinned vortex with a robust Majorana state. Phys. Rev. B 89, 163-167 (2014).

40. Sau, J. D., Lutchyn, R. M., Tewari, S. \& Das Sarma, S. Robustness of Majorana fermions in proximity-induced superconductors. Phys. Rev. B 82, 094522 (2010).
41. Chiu, C.-K., Gilbert, M. J. \& Hughes, T. L. Vortex lines in topological insulatorsuperconductor heterostructures. Phys. Rev. B 84, 144507 (2011).

42. Kawakami, T. \& Hu, X. Evolution of density of states and a spin-resolved checkerboard-type pattern associated with the majorana bound state. Phys. Rev. Lett. 115, 177001 (2015).

43. He, J. J., Ng, T. K., Lee, P. A. \& Law, K. T. Selective equal-spin Andreev reflections induced by Majorana fermions. Phys. Rev. Lett. 112, 037001 (2014).

44. Haim, A., Berg, E., von Oppen, F. \& Oreg, Y. Signatures of majorana zero modes in spin-resolved current correlations. Phys. Rev. Lett. 114, 166406 (2015).

45. Bode, M. Spin-polarized scanning tunnelling microscopy. Rep. Prog. Phys. 66, 523-582 (2003).

46. Wiesendanger, R. Spin mapping at the nanoscale and atomic scale. Rev. Mod. Phys. 81, 1495-1550 (2009).

47. Hu, L.-H., Li, C., Xu, D.-H., Zhou, Y. \& Zhang, F.-C. Theory of spin-selective Andreev reflection in the vortex core of a topological superconductor. Phys. Rev. B 94, 224501 (2016).

48. Sun, H. H. et al. Majorana zero mode detected with spin selective Andreev reflection in the vortex of a topological superconductor. Phys. Rev. Lett. 116, 257003 (2016).

49. Landau, L. A. et al. Towards realistic implementations of a Majorana surface code. Phys. Rev. Lett. 116, 050501 (2016).

50. Vijay, S., Hsieh, T. H. \& Fu, L. Majorana fermion surface code for universal quantum computation. Phys. Rev. X 5, 041038 (2015).

51. Vijay, S. \& Fu, L. Physical implementation of a Majorana fermion surface code for fault-tolerant quantum computation. Phys. Scripta T168, 014002 (2016).

Open Access This article is licensed under a Creative Commons Attribution 4.0 International License, which permits use, sharing, adaptation, distribution and reproduction in any medium or format, as long as you give appropriate credit to the original author(s) and the source, provide a link to the Creative Commons license, and indicate if changes were made. The images or other third party material in this article are included in the article's Creative Commons license, unless indicated otherwise in a credit line to the material. If material is not included in the article's Creative Commons license and your intended use is not permitted by statutory regulation or exceeds the permitted use, you will need to obtain permission directly from the copyright holder. To view a copy of this license, visit http://creativecommons org/licenses/by/4.0/.

(c) The Author(s) 2017 